\title{
Análise da produção científica dos programas de pósgraduação e seu alinhamento com as diretrizes do sistema nacional de ciência, tecnologia e inovação: um estudo cientométrico
}

\section{Analysis of the scientific production of graduate programs and its alignment with the guidelines of the national system of science, technology and innovation: a cientometric study}

\author{
Flávia Roberta Fernandes ${ }^{1}$, Helena de Fátima Nunes Silva ${ }^{1}$ \\ ${ }^{1}$ Universidade Federal do Paraná, Curitiba, PR, Brasil
}

Autor para correspondência/Mail to: Flávia Roberta Fernandes, flaroberta@gmail.com

Financiamento/Funding: Fundação Araucária e ao Governo do Estado do Paraná

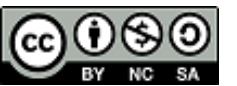

Copyright (C) 2018 Fernandes \& Silva. Todo o conteúdo da Revista (incluindo-se instruções, política editorial e modelos) está sob uma licença Creative Commons Atribuição-NãoComercial-Compartilhalgual 3.0 Não Adaptada. Ao serem publicados por esta Revista, os artigos são de livre uso em ambientes educacionais, de pesquisa e não comerciais, com atribuição de autoria obrigatória. Mais informações em http://revistas.ufpr.br/atoz/about/submissions\#copyrightNotice.

\begin{abstract}
Resumo
Os estudos e análises direcionados à produção científica e ao comportamento da ciência, atraem a atenção de órgãos governamentais, visto o subsídio de informações para a tomada de decisão, bem como a estruturação e o direcionamento de políticas públicas. Desta forma, os estudos bibliométricos e cientométricos, contribuem para a identificação do comportamento da ciência nas áreas específicas de estudo, assim como para a análise da produção e a utilização do conhecimento. Neste contexto, a presente pesquisa tem como objetivo analisar por meio da cientometria, a produção científica dos programas de pós-graduação, conceitos 6 e 7 e o alinhamento com as diretrizes do Sistema Nacional de Ciência, Tecnologia e Inovação (SNCTI). Para tanto, busca-se: (i) identificar a produção científica dos programas de pós-graduação, com conceitos 6 e 7; (ii) comparar a produção científica com as temáticas prioritárias e estratégicas do SNCTI (ENCTI 2016-2022); (iii) verificar os desdobramentos das produções científicas, a partir das temáticas prioritárias e estratégicas do SNCTI (ENCTI 2016-2022); (iv) examinar o perfil das produções científicas dos programas de pós-graduação; e ( $v$ ) identificar pesquisadores, grupos de pesquisa e redes de colaboração e a relação da produtividade e as temáticas prioritárias e estratégicas do SNCTI (ENCTI 2016-2022). A pesquisa configura-se como exploratória e descritiva, de abordagem quantitativa e qualitativa, com o delineamento por meio de pesquisa bibliográfica e documental. A coleta e o tratamento dos dados terão o suporte dos softwares Bibexcell e ScriptLattes. Os resultados serão pautados nos objetivos propostos e interpretados à luz do levantamento bibliográfico e documental. A partir das análises realizadas pretende-se avaliar a consonância das agendas de pesquisa dos programas de pós-graduação com as estratégias governamentais, seus desdobramentos e a contribuição da pesquisa para 0 fomento de planos, ações e o alinhamento dos focos de pesquisas institucionais.
\end{abstract}

Palavras-chave: Produção Científica. Sistema Nacional de Ciência, Tecnologia e Inovação. Cientometria.

\begin{abstract}
Research directed at scientific production and behavioral science draws attention of government agencies since it subsidizes information for decision making, and directs and structures public policies. In this way, bibliometric and scientometric research contribute to identify science behavior in specific areas of study, as well as production analysis and knowledge use. In this context, this research aims to analyze, through scientometric research, the scientific production of graduate programs with scores 6 and 7, and the alignment with the guidelines of the National System of Science, Technology and Innovation (SNCTI). In order to do so, this research aims to: (i) identify the scientific production of graduate programs, with scores 6 and 7; (ii) compare scientific production with priority and strategic themes of the SNCTI (ENCTI 2016-2022); (iii) verify the developments of scientific production, based on SNCTI's (ENCTI 2016-2022) priority and strategic themes; (iv) examine the profile of scientific production of graduate programs; and (v) identify researchers, research groups and collaboration networks, and productivity relationship. This research is characterized as descriptive and exploratory, with quantitative and qualitative approaches, with bibliographical and documental delineation. Data collection and processing will be supported by Bibexcell and ScriptLattes softwares. The results will be based on objectives proposed and interpreted in light of a bibliographical and documental survey. Through this research, it is intended to evaluate the consonance of graduate programs' research agendas with governmental strategies, its outcome and contribution to the promotion of plans, actions and alignment with the focus of institutional research.
\end{abstract}

Keywords: Scientific Production. National System of Science, Technology and Innovation. Scientometric.

\section{INTRODUÇÃO}

A informação e o conhecimento são "elementos cruciais" para o crescimento e avanços científicos, tecnológicos e econômicos (Castells, 1999, p. 119). Assim, o conhecimento produzido, a partir de demandas em áreas da sociedade e a "formulação e/ou implementação das metas nacionais de desenvolvimento" podem promover a aproximação entre a universidade, a pós-graduação e sociedade (Gazzola \& Fenati, 2010, p. 9). Segundo Danuello e Oliveira (2012), a concretização do conhecimento, por meio da produção científica, é utilizada como a principal ferramenta de mensuração e avaliação do desenvolvimento da ciência. No Brasil, em 2016 foram publicados um total de 68.908 artigos, entretanto, apesar do crescimento observado, ainda existe uma lacuna entre a produção científica brasileira e a dos países desenvolvidos em termos de produção científica e tecnológica, colocando-o em $14^{\circ}$ lugar no ranking mundial de produções científicas (Ministério da Ciência, Tecnologia e Inovações e Comunicações [MCTIC], 2016; Scimago, 2018). 
Neste sentido, a Estratégia Nacional de Ciência, Tecnologia e Inovação (ENCTI 2016-2022) estrutura-se visando a expansão, consolidação e interação das áreas de CTI (MCTIC, 2016) com vistas a promoção da pesquisa científica e dos programas de pós-graduação (stricto sensu) (MCTIC, 2016; Oliveira, 2015). Considerando tal aspecto, a mensuração de informações em áreas estratégicas e específicas, como forma de compreender o ambiente, o progresso e as tendências, pode utilizar a bibliometria e a cientometria como forma de análise (Santos, 2007). A cientometria pode ser considerada um mecanismo que contribui para análise e subsídio de informações para as tomadas de decisões estratégicas governamentais (Alvarez \& Caregnato, 2017), uma vez que a partir destas métricas, é possível ter uma visão ampla de cada área do conhecimento, redirecionar a alocação de recursos humanos, bem como direcionar fundos setoriais, principal instrumento do Governo Federal para fazer o Sistema de CTI crescer e avançar, tendo como prioridade investir em áreas de importância para o país (Financiadora de Estudos e Projetos [FINEP], 2018).

A produção científica e os respectivos estudos que analisam seus resultados, seus temas centrais (nesta pesquisa consideram-se como temas prioritários): (i) aeroespacial e defesa; (ii) água; (iii) alimentos; (iv) biomas e bioeconomias; (v) ciências e tecnologias sociais; (vi) clima; (vii) economia e sociedade digital; (viii) energia; (ix) minerais estratégicos; (x) nuclear; (xi) saúde; (xii) tecnologias convergentes e habilitadoras (MCTIC, 2016) e sua repercussão em cada área do conhecimento, podem ser considerados um instrumento que auxilia no direcionamento de estratégias para o desenvolvimento de políticas e diretrizes operacionais, da mesma forma que fornecem contribuições para um alinhamento das políticas e da condução da ciência e o impacto das pesquisas (Leite, 2010; Martins, Sandokhan, Silva, Oliveira, \& Silva, 2015), visto que as áreas de CTI "carecem de resultados avaliativos da produção científica brasileira que possam nortear investimentos e ações para melhoria da qualidade das investigações científicas nacionais" (Martins, 2002, p.1).

Neste contexto, a pesquisa propõe-se a responder a seguinte questão: Como os estudos cientométricos podem contribuir para a análise do alinhamento da produção científica dos programas de pós-graduação (conceitos 6 e 7) e as diretrizes do Sistema Nacional de Ciência, Tecnologia e Inovação.

Para isso, a pesquisa proposta será delineada a partir de um objetivo geral, sendo: analisar, por meio da cientometria, a produção científica dos programas de pós-graduação (6 e 7) e o alinhamento com as diretrizes do Sistema Nacional de Ciência, Tecnologia e Inovação (SNCTI) e cinco específicos: (i) identificar a produção científica dos programas de pós-graduação, com conceitos 6 e 7; (ii) comparar a produção científica com as temáticas prioritárias e estratégicas do SNCTI (ENCTI 2016-2022); (iii) verificar os desdobramentos das produções científicas, a partir das temáticas prioritárias e estratégicas do SNCTI (ENCTI 2016- 2022); (iv) examinar o perfil das produções científicas, dos programas de pós- graduação; e (v) identificar pesquisadores, grupos de pesquisa e redes de colaboração e a relação da produtividade e as temáticas prioritárias e estratégicas do SNCTI (ENCTI 2016-2022).

A pesquisa justifica-se pela contribuição analítica de informações estratégicas, permitindo articular e direcionar os esforços dos atores envolvidos no SNCTI. Concomitantemente, os estudos cientométricos e bibliométricos, juntamente com suas análises despertam o interesse de setores do governo pelo subsídio de informações para a tomada de decisão e direcionamento de políticas, nas áreas de CTI (Alvarez \& Caregnato, 2017; Macias-Chapula, 1998; Hayashi, 2012; Machado, 2007; Santos \& Kobashi, 2009; Silva, Hayashi, \& Hayashi, 2011), assim como para o direcionamento de recursos humanos e financeiros (Silva \& Biachi, 2001).

Do ponto de vista institucional, a pesquisa pode contribuir com outros estudos, tanto para a Universidade Federal do Paraná (UFPR), quanto para as outras universidades em termos de indicar áreas de pesquisas prioritárias, assim como a "apropriação desses indicadores por parte de órgãos financiadores pode resultar na abertura de cursos de pós-graduação e na formação de novo capital humano" (Alvarez \& Caregnato, 2017, p. 23). O estudo está em consonância com o Programa de Pós-Graduação em Gestão da Informação (PPGGI) e com a linha de pesquisa Informação, Conhecimento e Estratégia, uma vez que concentra pesquisas pautadas nos processos de informação e do conhecimento como forma de subsidiar a tomada de decisão. Da mesma forma que as discussões teóricas promovidas, bem como o método empregado, contribuirão para outras pesquisas. A originalidade da pesquisa, envolve a escolha do foco de análise, visto que não foram localizados estudos, no Banco de Dissertações e Teses (Instituto Brasileiro de Informação em Ciência e Tecnologia [IBICT], 2018; Capes, 2018), que se proponham a realizar a comparação da produção científica com as estratégias prioritárias na área de CTI (ENCTI 2016-2022). Desta forma, o estudo pode contribuir para promover o conhecimento em áreas específicas, o direcionamento de recursos, conduzir análises em diferentes perspectivas, alavancar os resultados das áreas e atores envolvidos, subsidiar o governo com informações que permitam melhor posicionamento do Brasil entre os países referência na área (MCTIC, 2016), "identificar os focos produtores do conhecimento novo, como sua elite científica" (Castanha \& Grácio, 2012, p. 83) frente as demandas de pesquisa apontadas como prioritárias em âmbito nacional e internacional, além de fornecer informações para o direcionamento de fundos setoriais (FINEP, 2018). O bom uso dos recursos de telecomunicações nas atividades de segurança pública podem representar para o Estado, o controle da informação e assim, na visão de Braman (2006) reforçar o papel do Estado na manutenção do poder informacional. 


\section{REVISÃO DE LITERATURA}

A revisão de literatura se fundamentará em trabalhos, a partir dos autores seminais e pesquisas na esfera nacional e internacional sobre informação estratégica, produção científica e cientometria. Entretanto, neste pré-projeto são apresentados apenas os principais conceitos de cientometria e a produção científica.

De acordo com Kuhn (1989), a ciência, reúne fatos, métodos e teorias sistematizados em texto e caracteriza-se pela divulgação dos resultados de uma pesquisa, permitindo que novos conhecimentos sejam produzidos (Chan, Okune, \& Sambuli, 2015). A produção científica é considerada a geração de conhecimento por meio da pesquisa (Leite, 2010; Silva et al., 2011) e abrange a comunicação dos resultados dos estudos realizados (Silva, Menezes, Pinheiro, \& Schweitzer, 2006). Da mesma forma, pode ser apontada como produto da atividade científica (Silva et al, 2006), materializada por meio de teses, dissertações, artigos, livros, comunicações em eventos, entre outros (Silva et al., 2011), sendo vista como uma ferramenta de análise da ciência (Danuello \& Oliveira, 2012). As produções promovem o reconhecimento do pesquisador por seus pares e pela comunidade acadêmica (Morel \& Morel, 1977) subsidiam informações para questões e área específicas, atualizam informações e apresentam tendências nas áreas estudadas (Droescher \& Silva, 2014). O aumento das produções científicas tem como fatores de influência, a formação de mestres e doutores (Leite, 2010), assim como a consolidação e expansão das áreas de CTI (Rezende, 2011).

A pesquisa e a produção científica são insumo para o avanço mundial (Droescher \& Silva, 2014) e neste quesito, os estudos da ciência "os indicadores bibliométricos e cientométricos tornam-se essenciais" (Macias-Chapula, 1998, p. 134), visto que são capazes de medir a informação (Hayashi, 2012). As técnicas são utilizadas para analisar produções científicas (bibliometria) e para estudar as atividades da ciência (cientometria) (Hayashi, 2012; Santos \& Kobashi, 2009). Segundo Hayashi (2012), os indicadores bibliométricos e cientométricos evoluíram a partir de 1960, com a demanda de avaliar as áreas de CTI. Visto que ambas as técnicas procuram estudar quantitativamente a ciência, a geração e circulação da produção científica (Hayashi, 2012; Santos \& Kobashi, 2009; Vasconcelos, 2014), bem como sua utilização permite mensurar os avanços científicos (Macias-Chapula, 1998; Silva \& Biachi, 2001), mapear o "campo científico e extrair informações úteis para a compreensão de sua estrutura social e intelectual" (Hayashi, 2012, p. 26). A distinção entre a bibliometria e a cientometria encontra-se no enfoque do objeto de estudo, sendo uma voltada para livros e revistas (bibliometria) e a outra para a circulação e consumo das produções (cientometria) (Santos \& Kobashi, 2009; Vasconcelos, 2014).

Para a realização das análises bibliométricas, três leis são utilizadas: (i) Lei de Lotka para a produtividade científica; (ii) Lei de Bradford para a dispersão da produção científica; e (iii) Lei de Zipf, relacionando a ocorrência de palavras no texto (Bufrem, 2005; Guedes, 2012). A análise de citações, "em gêneros textuais acadêmicos, é um dos métodos mais utilizados principalmente para a tomada de decisão na política científica e tecnológica" (Guedes, 2012, p. 101). As leis permitem analisar e descrever aspectos da literatura pesquisada, como: abordagem do autor, linha de pensamento, enfoque de pesquisa, contribuições referenciadas pelos pares, frequência de citações, exploração de conceitos, perfil dos pesquisadores, interesses de pesquisa, dentre outros (Vasconcelos, 2014). A partir destas análises, as informações levantadas podem subsidiar orientações para a tomada de decisão quanto a dinâmica da ciência nacional e sua relação com a ciência mundial (Macias-Chapula, 1998).

\section{PROCEDIMENTOS METODOLÓGICOS}

A pesquisa define-se como exploratória, visto que busca ampliar o arcabouço teórico sobre o tema de pesquisa e descritiva, pois pretende investigar fenômenos e suas relações com o objeto pesquisado (Vieira, 2009). A abordagem caracteriza-se como mista (quantitativa e qualitativa), por pautar-se na utilização de estudos cientométricos, que permitem a mensuração e análise estatísticas dos dados e qualitativa, uma vez que utilizará de análise de conteúdo para analisar as produções científicas (Vieira, 2009). O delineamento da pesquisa se dará por meio de pesquisa bibliográfica e pesquisa documental (Flick, 2009).

A população se refere a um total de 4.175 Programas de Pós-Graduação (stricto sensu), divididos em: Doutorado (74), Mestrado (1.270), Mestrado Profissional (703) e Mestrado e Doutorado (2.128) (Capes, 2017). A amostra desta pesquisa é intencional (Mensure Evaluation, 2016), pois optou-se pelos programas considerados de excelência e avaliados com conceitos 6 e 7, por se aproximarem de critérios internacionais de produção científica (Coordenação de Aperfeiçoamento de Pessoal de Nível Superior [Capes], 2017). Do total de 4.175 programas, 483, enquadram-se entre os programas de excelência, de acordo com as 9 "Grandes áreas" de avaliação (Capes, 2017b), sendo: Ciências Agrárias (56), Ciências Biológicas (72), Ciências da Saúde (79), Ciências Exatas e da Terra (64), Engenharia (52), Multidisciplinar (34), Ciências Humanas (55), Ciências Sociais Aplicadas (44) e Linguística, Letras e Artes (27), sendo estes os que comporão a amostra de pesquisa.

O delineamento da pesquisa se dará por meio de: (i) levantamento bibliográfico, tendo como apoio a consulta as bases de dados referência na área de Ciência da Informação, conforme breve levantamento já realizado nas bases da Web of Science e SciElo ${ }^{1}$, e em produções referenciadas nestes artigos levantados (Quadro 1); e (ii) pesquisa

${ }^{1}$ Web Of Science (http://app- webofknowledge.ez22.periodicos.capes.gov.br/) e SciElo (http://www.scielo.org) 
documental, a partir de bases de dados governamentais ${ }^{2}$.

\begin{tabular}{|c|c|}
\hline Conceito & Autores Internacionais e Nacionais \\
\hline $\begin{array}{l}\text { Informação e } \\
\text { estratégia }\end{array}$ & $\begin{array}{l}\text { B. Kirk (1999); Citroen (2011); Davenport (1998, 2004); Detlon (2010); Drnevich (2013); } \\
\text { Fidelis (2006); Fadel et. al (2010); Fidelis e Barbosa (2012); Leitão (1993); McGee e Prusak } \\
\text { (1994); Mintzberg (2010); Paletta e Mansold (2016); Porter (1999), Valentim (2002). }\end{array}$ \\
\hline $\begin{array}{l}\text { Produção ci- } \\
\text { entífica }\end{array}$ & $\begin{array}{l}\text { Abbot et al. (2010); Almeida e Guimarães (2010); Alvarenga (2000); Batovski (2008); Bunge } \\
\text { (1980); Castro (2005); Crespo e Caregnato (2004); Abdo (2015); Chan, Okune e Sambuli } \\
\text { (2015); Garvey (1979); Gibbons et al. (1994); Guimarães e Human (1995); Hahn (2008); } \\
\text { Meadows (1999); Miranda (1998); Muller (1995); Población e Oliveira (2006); Price (1963; } \\
\text { 1976); Sola Price (1976); Strehl, Calabró e Amaral (2016); Targino (2000); Viotti (2003); Ziman } \\
\text { (1979). }\end{array}$ \\
\hline Cientometria & $\begin{array}{l}\text { Abramo e DÁngelo (2011); Araújo (2006); Bianchi (2001); Edge (1977); Garfield (1979, 1985, } \\
\text { 1996); Guedes (2012); Hayashi (2012)); Santos e Kobashi (2009); Leydesdorff (1998, 2001, } \\
\text { 2005); Machado (2007); Macias-Chapula (1998); Maricato (2011); Mingers e Leydesdorff } \\
\text { (2015); Mugnani (2006); Otlet (1934); Pritchard (1969); Spinak (1996, 1998); Urbizagastegui } \\
\text { (2008); Vanti (2002, 2005, 2011); Vanz e Stumpf (2010); Vasconcelos (2014); Wormell (1998); }\end{array}$ \\
\hline
\end{tabular}

Quadro 1. Conceitos, abrangência e autores

Fonte: Elaborado pelas autoras a partir das bases de dados SciElo e Web of Science (2018).

Por uma limitação da quantidade de páginas para a apresentação deste projeto, optou-se por apresentar, as etapas da coleta e análise de dados, por meio de uma representação gráfica, conforme Figura 1.

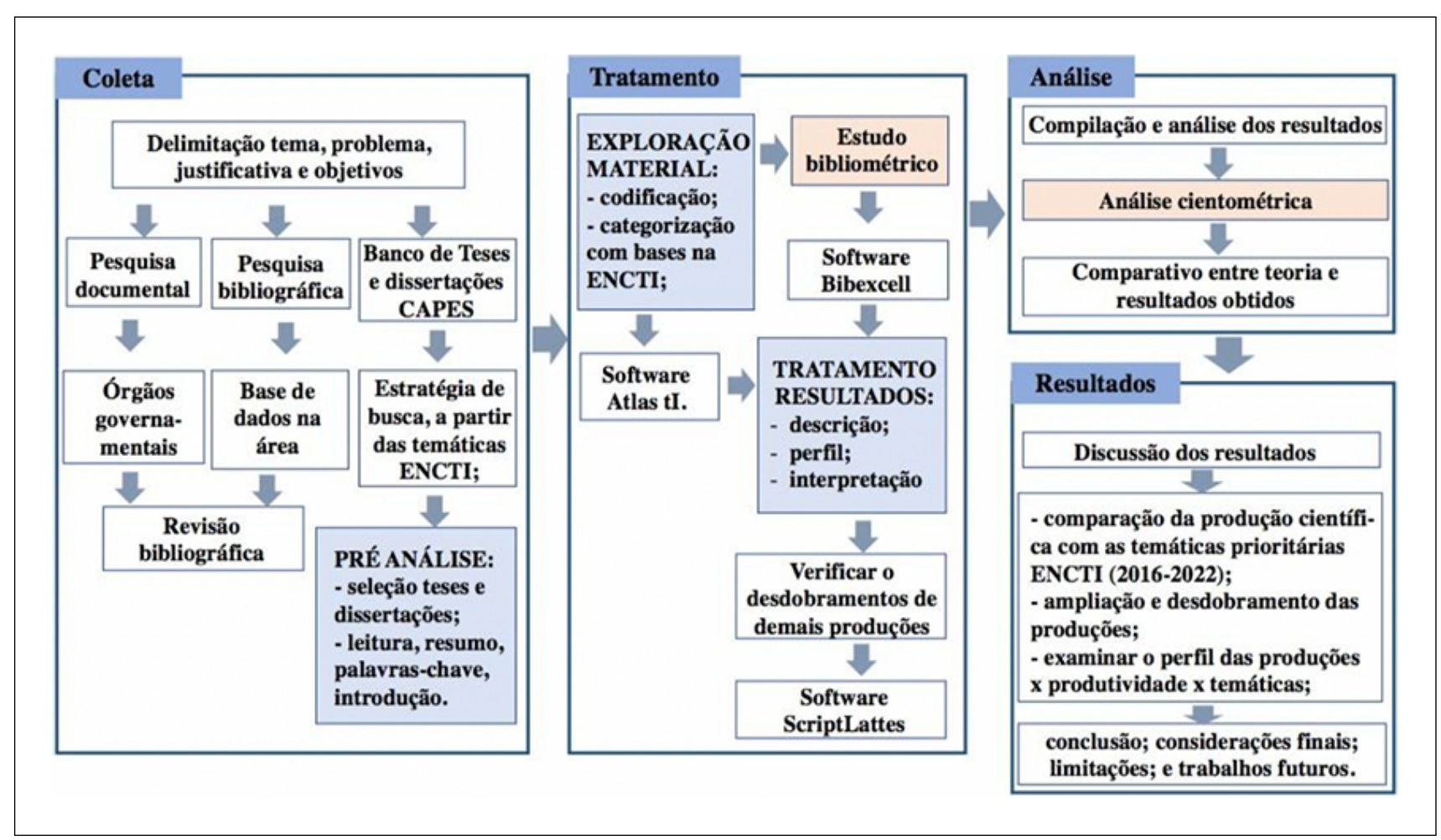

Figura 1. Etapas da coleta, análise de dados e resultados Fonte: Elaborado pelas autoras

\section{CONSIDERAÇÕES PARCIAIS}

A análise dos resultados será pautada nos objetivos específicos, no levantamento empírico, bem como interpreta a partir do levantamento bibliográfico e documental. Com a observação das produções científicas nos programas de pós- graduação brasileiros com conceitos 6 e 7, pretende-se verificar se as agendas de pesquisas da pós-graduação estão em consonâncias com as necessidades governamentais de desenvolvimento, bem como avaliar as contribuições das áreas específicas para o atingimento do objetivo nacional e posicionamento internacional (MCTIC, 2016).

A comparação entre a produção científica com as temáticas prioritárias e estratégicas do SNCTI (ENCTI 2016-2022) permitirá verificar dentro das "grandes as áreas do conhecimento" os programas, docentes e pesquisas

\footnotetext{
${ }^{2}$ Bases governamentais do Ministério da Ciência, Tecnologia, Inovação e Comunicação (MCTIC), da Coordenação de Aperfeiçoamento de Pessoal de Nível Superior (Capes), do Conselho Nacional de Desenvolvimento Científico e Tecnológico (CNPq), entre outros.
} 
que estão sendo voltadas para as demandas da sociedade e atenção do governo. Os desdobramentos das produções científicas a partir das temáticas prioritárias e estratégicas do SNCTI (ENCTI 2016-2022) permitirão uma ampliação da investigação em termos de produção dos programas.

Por fim, examinar o perfil/comportamento das produções científicas (programas, pesquisadores, grupos de pesquisa, redes de colaboração versus produtividade) em comparação com a produtividade e as temáticas prioritárias e estratégicas do SNCTI (ENCTI 2016-2022), permitirá verificar o desenvolvimento das metas, aprimorar e fomentar políticas e diretrizes setoriais, o desenvolvimento de proposição de demais programas, planos e ações, bem como alinhar a proposição e o foco nas agendas de pesquisas institucionais.

\section{AGRADECIMENTOS}

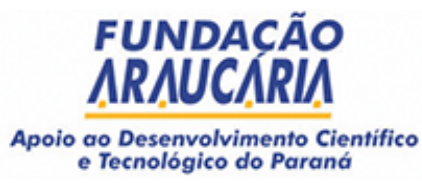

FUNDACÃO

Apoio ao Desenvolvimento Cientifico

e Tecnológico do Paran

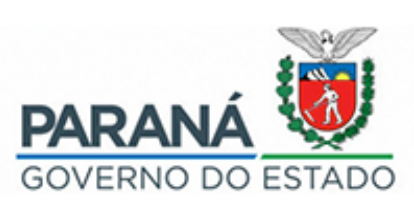

À Fundação Araucária e ao Governo do Estado pelo apoio financeiro para a realização do evento.

\section{REFERÊNCIAS}

Alvarez, G. R., Caregnato, S. E., \& Caregnato, S. E. (2017). A ciência da informação e sua contribuição para a avaliação do conhecimento científico. Biblos: Revista Do Instituto de Ciências Humanas e Da Informação, 31(1), 09-26. Recuperado de https://doi.org/10.14295/biblos.v31i1.5987

Braman, S. (2006). Change of State: information, policy, and power. Cambridge Mand London: MIT Press.

Bufrem, L. (2005). O saber científico registrado e as práticas de mensuração de informação. Ci. Inf., 34(2), 9-25. Recuperado de http://www.scielo.br/pdf/ci/v34n2/28551

Castanha, R. C. G., \& Grácio, M. C. C. (2012). Indicadores de avaliação de Programas de Pós-Graduação: um estudo comparativo na área da Matemática. Em Questão, 18(3), 81-97, Recuperado de https://seer.ufrgs.br/Em Questao/article/view/33192

Castells, M. (1999). A Sociedade Em Rede: Economia, Sociedade e Cultura. São Paulo: Paz e Terra.

Chan, L., Okune, A., \& Sambuli, N. (2015). O Que é Ciência Aberta E Colaborativa E Que Papéis Ela Poderia Desempenhar No Desenvolvimento? In S. Albagli, M. L. Maciel, \& A. H. Abdo. (2015). Ciência aberta, questões abertas. Rio de Janeiro: UNIRIO.

Coordenação de Aperfeiçoamento de Pessoal de Nível Superior. Portaria n. 59, de 22 de março de 2017 (2017). Brasil. Recuperado de https://www.capes.gov.br/images/stories/download/legislacao/27032017PORTARIA-N-59-DE-22-DE-MARCO-DE-2017.pdf

Coordenação de Aperfeiçoamento de Pessoal de Nível Superior. Resultado da Avaliação Quadrienal 2017 Resultados finais por área programas acadêmicos. (2017). Recuperado em 13 de fevereiro, 2018, de http://avaliacaoquadrienal.capes.gov.br/resultado-da-avaliacao-quadrienal-2017-2

Coordenação de Aperfeiçoamento de Pessoal de Nível Superior. Catálogo de teses e dissertações. (2018). Recuperado de http://catalogodeteses.capes.gov.br/catalogo-teses/\#!/

Danuello, J. C., \& Oliveira, E. F. (2012). Análise cientométrica: produção científica e redes colaborativas a partir das publicações dos docentes dos programas de pós-graduação em Fonoaudiologia no Brasil. Em Questão, 18(3), 65-79. Recuperado de https://seer.ufrgs.br/index.php/EmQuestao/article/view/33178

Droescher, F. D., \& Silva, E. L. da. (2014). O pesquisador e a produção científica. Perspectivas Em Ciência Da Informação, 19(1), 170-189. Recuperado de https://doi.org/10.1590/S1413-99362014000100011

Financiadora de Estudos e Projetos (FINEP). O que são fundos setoriais. (2018). Recuperado de http://www.finep. gov.br/a-finep-externo/fontes-de-recurso/fundos-setoriais/o-que-sao-fundos-setoriais

Flick, U. (2009). Introdução à pesquisa qualitativa (3a ed.). Porto Alegre: Artmed.

Gazzola, A. L. A., \& Fenati, R. (2010). A PG Brasileira no ano de 2020. In Ministério da Educação. Coordenação de Aperfeiçoamento de Pessoal de Nível Superior. Plano Nacional De Pós-Graduação - PNPG 2011-2020: documentos setoriais. Brasília. Recuperado de https://capes.gov.br/images/stories/download/PNPG_Miol o_V2.pdf 
Guedes, V. L. (2012). A Bibliometria e a gestão da informação e do conhecimento científico e tecnológico: uma revisão da literatura. PontodeAcesso, 6(2), 74-109. Recuperado de https://portalseer.ufba.br/index.php/revista ici/article/view/5695

Hayashi, M. C. (2012). Sociologia da ciência, bibliometria e cientometria: contribuições para a análise da produção científica. In Anais eletrônicos do Seminário de Epistemologia e Teorias da Educação. Recuperado de https://www.marilia.unesp.br/Home/Graduacao/PETBiblioteconomia/soc-da-ciencia-pet.pdf

Instituto Brasileiro de Informação em Ciência e Tecnologia (2018). Biblioteca Digital Brasileira de teses e dissertações. Recuperado de http://bdtd.ibict.br/vufind/

Kuhn, T. A. (1989). Estrutura das Revoluções Científicas. São Paulo: Perspectiva.

Leite, G. A., Filho. (2010). Perfil da produção científica dos docentes e programas de pós- graduação em ciências contábeis no Brasil. Revista de Contabilidade e Controladoria, 2(2), p. 1-13. Recuperado de https://revistas.ufpr.br/rcc/article/view/19370

Machado, R. N. (2007). Análise cientométrica dos estudos bibliométricos publicados em periódicos da área de biblioteconomia e ciência da informação (1990-2005). Perspectivas em Ciência da Informação, 12(3), 2-20. Recuperado de http://portaldeperiodicos.eci.ufmg.br/index.php/pci/article/view/144

Macias-Chapula, C. A. (1998). O papel da informetria e da cienciometria e sua perspectiva nacional e internacional. Ci. Inf., 27(2), 134-140. Recuperado de http://www.scielo.br/pdf/ci/v27n2/macias.pdf

Martins, D. L., Sandokhan, R., Silva, A., Oliveira, L. F. R. de, \& Silva, E. A. (2015). Mapeando as correlações entre produtividade e investimentos de bolsas em programas de pós-graduação: o caso da Universidade Federal de Goiás. Em Questão, 21(2), 162. Recuperado de https://doi.org/10.19132/1808-5245212.162-180

Martins, G. A. Divulgação de trabalho: considerações sobre os doze anos do caderno de estudos. Rev. Contab. Finanç., 13(30), p. 81-88. Recuperado de http://dx.doi.org/10.1590/S1519-70772002000300007

Ministério da Ciência, Tecnologia e Inovações e Comunicações. (2016). Estratégia Nacional de Ciência, Tecnologia e Inovação 2016 - 2022. Brasília: MCTIC. Recuperado de http://www.finep.gov.br/images/afinep/Politica/16_03_2018_Estrategia_Nacional_de_Ciencia_Tecnologia_e_Inovacao_2016_2022.pdf

Morel, R. L. M, \& Morel, C. M. (1977). Um estudo sobre a produção científica brasileira, segundo os dados do institute for scientific information (ISI). Ci. Inf. Rio de Janeiro, 6(1), p. 99-109. Recuperado de http://revista.ibict.br/ciinf/article/view/85

Oliveira, J. F. de. (2015). A pós-graduação e a pesquisa no Brasil: processos de regulação e de reconfiguração da formação e da produção do trabalho acadêmico. Práxis Educativa, 10(2), p. 343-363. Recuperado de http://www.revistas2.uepg.br/index.php/praxiseducativa/article/view/7138

Rezende, S. M. (2011). Produção científica e tecnológica no Brasil: conquistas recentes e desafios para a próxima década. Revista de Administração de Empresas, 51(2), 202-209. Recuperado de https://doi.org/10.1590/s00 34-75902011000200007

Santos, P. (2007). Paul Otlet: um pioneiro da organização das redes mundiais de tratamento e difusão da informação registrada. Ciência da Informação, (36) 2, p. 54-63. Recuperado de http://www.scielo.br/scielo.php?pid $=$ S0100-19652007000200006\&script $=$ sci_abstract\&tlng $=$ pt

Santos, R. N. M., \& Kobashi, N. Y. (2009). Bibliometria, cientometria, informetria: conceitos e aplicações. Pesq. Bras. Ci. Inf., 2(1), p.155-172. Recuperado de https://repositorio.ufpe.br/handle/123456789/10089

Scimago. SJR-SCImago Journal \& Country Rank. (2018). Recuperado de http://www.scimagojr.com

Silva, E. L. da, Menezes, E. M., Pinheiro, L. V., \& Schweitzer, F. (2006). Panorama da pesquisa em Ciência da Informação no Brasil. Informação \& Sociedade: Estudos, 16(1). Recuperado de http://www.periodicos.ufpb.br /ojs/index.php/ies/article/view/451/0

Silva, J. A., \& Bianchi, M. L. P. (2001). Cientometria: a métrica da ciência. Paidéia (Ribeirão Preto), 11(21), p. 5-10. Recuperado de http://dx.doi.org/10.1590/S0103-863X2001000200002

Silva, M. R., Hayashi, C. R. M., \& Hayashi, M. C. P. I. (2011). Análise bibliométrica e cientométrica: desafios para especialistas que atuam no campo. In CID: R. Ci. Inf. e Doc., 2(1), p. 110-129. Recuperado de https://doi.org/10.11606/issn.2178-2075.v2i1p110-129

Vasconcelos, Y. L. Estudos Bibliométricos: procedimentos metodológicos e contribuições. Ciências Jurídicas, 15(2), p. 211-220. Recuperado de http://revista.pgsskroton.com.br/index.php/juridicas/article/view/307

Vieira, S. (2009). Como elaborar questionários. São Paulo: Atlas. 